\title{
Interplay of foot-and-mouth disease virus, antibodies and plasmacytoid dendritic cells: virus opsonization under non-neutralizing conditions results in enhanced interferon-alpha responses
}

\author{
Nils Lannes, Sylvie Python and Artur Summerfield ${ }^{*}$
}

\begin{abstract}
Foot-and-mouth disease virus (FMDV) is a highly infectious member of the Picornaviridae inducing an acute disease of cloven-hoofed species. Vaccine-induced immune protection correlates with the presence of high levels of neutralizing antibodies but also opsonising antibodies have been proposed as an important mechanism of the immune response contributing to virus clearance by macrophages and leading to the production of type-I interferon (IFN) by plasmacytoid dendritic cells (pDC). The present study demonstrates that the opsonising antibody titres mediating enhanced IFN-a responses in $\mathrm{pDC}$ were similar to neutralizing titres, when antigenically related viruses from the same serotype were employed. However, sera cross-reacted also with non-neutralized isolates of multiple serotypes, when tested in this assay. Both uncomplexed virus and immune complexed virus stimulated pDC via Toll-like receptor 7. An additional finding of potential importance for strain-specific differences in virulence and/or immunogenicity was that pDC activation by FMDV strongly differed between viral isolates. Altogether, our results indicate that opsonising antibodies can have a broader reactivity than neutralizing antibodies and may contribute to antiviral responses induced against antigenically distant viruses.
\end{abstract}

\section{Introduction}

Foot-and-mouth disease virus (FMDV) is a highly contagious infectious agent inducing disease of cloven-hoofed animals including cattle, swine, goats and sheep. Due to the significant economic impact on livestock, a tight disease control is required. However, its high mutation rate contributes to immune escape and the presence of seven serotypes (O, A, C, Asia-1, South African Territories 1, 2 and 3) each containing a large variety of isolates with high antigenic variability.

Current conventional vaccines, consisting of inactivated virus, provide a short-term serotype specific protection. However, vaccination does not induce protection against all isolates within one serotype [1]. Protection is related to the presence of high level of neutralizing antibody in serum. However, animals with low levels of neutralizing antibodies can also be protected $[2,3]$.

\footnotetext{
* Correspondence: artur.summerfield@ivi.admin.ch

Institute of Virology and Immunoprophylaxis, Sensemattstrasse 293, 3147, Mittelhäusern, Switzerland
}

Furthermore, non-neutralizing concentrations of monoclonal antibodies $(\mathrm{mAb})$ can induce protection in mice [4]. Thus, other mechanisms than neutralization could be involved in protection. It has been shown that opsonisation of FMDV enhances phagocytosis by monocytes and macrophages in vitro [5]. More recent in vivo data emphasize the potential role of opsonising antibodies in a mouse model, in which protection was mediated in a macrophage-dependent manner [6]. While these studies indicate that immune complexed virus could be eliminated after phagocytosis by macrophages bearing Fc receptors $(\mathrm{FcR})$, other studies also indicate a participation of dendritic cells (DC), at least in vitro. Immune complexes induce the activation of porcine plasmacytoid DC (pDC) resulting in the production of interferon (IFN)- $\alpha$ [7]. The possible involvement of opsonising antibodies in FMDV immunity was also confirmed with bovine pDC [8].

PDC represent $0.1-0.5 \%$ of porcine peripheral blood mononuclear cells (PBMC), with similar functional 
characteristics to their human and murine counterparts [9]. They are characterized by producing high amount of antiviral type-I IFN in response to a wide range of pathogens. pDC activation is mediated by sensing pathogen-associated molecular patterns (PAMP) through pattern recognition receptors (PRR). By expressing PRR, such as Toll-like receptors (TLR) and C-type lectin receptors, $\mathrm{pDC}$ contribute to the innate and adaptive antiviral immunity [10]. Antibody-dependent FMDV entry in pDC occurs via the Fc $\gamma$ RII receptor (CD32) [7], linking $\mathrm{pDC}$ to the adaptive immunity [11].

Considering the possible importance of opsonising antibodies and $\mathrm{pDC}$ in the protection against FMDV, the main aim of this study was to characterize the relationship between neutralizing and opsonising activities of polyclonal sera from immunized pigs. Although neutralization and opsonisation occurred at similar serum dilutions when antigenically related viruses were employed, opsonisation also occurred in the absence of neutralization and across different serotypes. We also discovered differences in the ability of various FMDV isolates to activate pDC.

\section{Materials and methods}

Antibodies and phenotyping

For pDC enrichment, monoclonal antibodies against following cell surface markers were used: CD172a (mAb 7422-15A), CD14 (mAb CAM36A), CD3 (mAb 8E6) and CD4 (mAb PT90A). For phenotyping, mAb against CD172a and CD4 were used. Hybridoma for mAb 74-22$15 \mathrm{~A}$ was kindly provided by Dr A. Saalmüller (Veterinary University, Vienna, Austria). mAbs CAM36A, 8E6 and PT90A were purchased from VMRD (Pullman, WA, USA).

\section{Cell culture}

Unsorted and sorted (see below) PBMC were cultured in Dulbecco's modified Eagle's minimal essential medium (DMEM) plus GlutaMAX '-I (GIBCO, Life Technologies, Basel, Switzerland) supplemented with $20 \mu \mathrm{M}$ of $\beta$ mercaptoethanol (Life Technologies) at $39^{\circ} \mathrm{C}$ at $6 \% \mathrm{CO}_{2}$. Baby Hamster Kidney (BHK) 21 cells were grown in Glasgow's minimum essential medium (GMEM, Life Technologies) supplemented with 5\% v/v Fetal Bovine Serum (FBS, South America Origin, Biowest, Nuaillé, France). For virus preparation and serum neutralization test, cells were cultured in FBS-free GMEM at $37^{\circ} \mathrm{C}, 6 \% \mathrm{CO}_{2}$.

\section{Enrichment of pDC and purity check}

Blood was collected alternatively from a total of 10 specific pathogen-free (SPF) pigs of 2-24 months old kept at our institute. PBMC were isolated from citrated blood using Ficoll Paque (1.077 g/L, Amersham Pharmacia Biotech AG, Dubendorf, Switzerland) density gradient [12]. For pDC enrichment, PBMC were separated using magnetic sorting system (MACS) with depletion (LD) and selection (LS) columns (Miltenyi Biotech GmbH, Bergisch-Gladbach, Germany). pDC were enriched either using CD172a positive selection with LD columns or by a first depletion of $\mathrm{CD} 14^{+}$cells with a subsequent positive selection for $\mathrm{CD} 172 \mathrm{a}^{+}$cells. Alternatively, PBMC were isolated using Ficoll Paque and Optiprep (60\% w/v solution of oidixanol in water, Sigma-Aldrich, Saint Louis, MO, USA) density gradients followed by a depletion of $\mathrm{CD}^{+}$cells and a final enrichment of $\mathrm{CD} 4^{+}$cells [13]. Purity of the sorted population was verified by flow cytometry detection, after staining with anti-CD172a and anti-CD4 mAbs and isotype-specific R-phycoerythrin (R-PE) and fluorescein isothiocyanate (FITC) conjugates (Southern Biotechnology Associates, Birmingham, AL, USA) as described [14]. The pDC population was identified as $\mathrm{CD} 4{ }^{\text {high }} \mathrm{CD} 172 \mathrm{a}^{\text {low }}$ cells by flow cytometry [15].

\section{Virus preparation}

Isolates of FMDV were propagated in BHK-21 cells as previously described [16] and viral titres were determined by end-point titration on BHK-21 cells [5]. O UKG 2001, C1 Noville, O Bulgaria 1/91, O VietNam $7 /$ 97, A Brazil 10/93, A Turkey/99 and Asia-1 Turkey/99 were kindly provided by Drs. Nigel Ferris and Satya Parida (Institute for Animal Health, Pirbright, UK). In order to avoid heparin sulphate adaptation of the virus, the isolates were not passaged more than three times in BHK-21 cells [17]. Mock antigen was prepared from uninfected BHK-21 cells in the same manner as FMDV.

\section{Production of immune serum}

Two SPF pigs were immunized intra-muscularly with the full-dose of a monovalent FMDV vaccine consisting of inactivated type-O Manisa antigen (Merial, Lyon, France). Animals received prime injection at day 0 and a booster at day 21 . At day $0,14,21,28$, and 42, blood samples were taken and serum prepared for storage at $-20^{\circ} \mathrm{C}$. Serum samples from a naïve animal of the same litter were used as controls. In certain experiments, serum was complement inactivated by heat-treatment at $56^{\circ} \mathrm{C}$ for $30 \mathrm{~min}$.

\section{Serum neutralization test}

Serial dilutions of heat-treated serum were incubated at room temperature (RT) for 30 min with $100 \mathrm{TCID}_{50}$ of FMDV isolate to form immune complexes in final volume of $100 \mu \mathrm{L} /$ well and then added to confluent BHK21 cells, grown in 96-well plate for 3 days. The neutralization titre was calculated according to Kaerber [18] based on FMDV-induced cytopathogenic effect (CPE). 


\section{Induction of type-I IFN and inhibition of the TLR7 pathway}

Isolated PBMC and sorted cells $\left(4 \times 10^{6}\right.$ cells $\left./ \mathrm{mL}\right)$ were stimulated in $100 \mu \mathrm{L}$ of serum-free medium with FMDV, FMDV/immune serum mixture or FMDV/naïve serum mixture. Sera were used either untreated or heat-treated. FMDV/serum mixtures were previously formed for $30 \mathrm{~min}$ at $39^{\circ} \mathrm{C}$. As control, cells were stimulated with CpG D32 (10 $\mu \mathrm{g} / \mathrm{mL}$, Invitrogen, Basel, Switzerland). Influenza virus strain PR8 was employed to test the specificity of the TLR7 inhibitor IRS661 (5'-TGCTTGCAAGCTTGCAAGCA-3'). As control, the oligonucleotide (ODN) sequence (5'TCCTGCAGGTTAAGT-3') was used [19]. IRS661 and Ctrl-ODN were purchased from Eurofins MWG Operon (Ebersberg, Germany). Influenza virus was grown on 10 days embryonated chicken eggs and titrated as previously described [20]. pDC activation by influenza virus employed an multiplicity of infection (MOI) of $10 \mathrm{TCID}_{50} /$ cell [21]. Supernatants from enriched $\mathrm{pDC}$ were harvested after $24 \mathrm{~h}$ and IFN- $\alpha$ was detected by specific ELISA as described [14]. Data were collected using a VersaMax photometer with SOFTmax Pro software.

\section{Statistical analysis}

Significant differences were determined with SigmaPlot v11 using the Sum Rank Test $(P<0.005)$.

\section{Results}

\section{Impact of enrichment method on pDC activation by FMDV}

We previously documented that $\mathrm{pDC}$ activation by FMDV occurs only in presence of specific antibodies [7]. However, these experiments were performed with $\mathrm{pDC}$, which were only enriched about $\sim 5-10$ fold from PBMC using a CD172a positive selection by magnetic cell sorting (Figure 1A). Therefore, more efficient purification methods were evaluated in this study. $\mathrm{pDC}$ are non-T cells (CD3 ) [22] and are found within the CD172a${ }^{\text {low }} \mathrm{CD} 4{ }^{\text {high }} \mathrm{CD} 14$ PBMC [15]. Based on this, PBMC were first depleted from CD14 $4^{+}$monocytes and subsequently enriched using CD172a. This resulted in a frequency of $3-5 \%$ of pDC ( 20 fold enrichment), depending on the experiment. A third protocol employed a CD3 depletion and subsequent CD4 enrichment of PBMC resulting in a pDC population of 15-60\% (Figure 1A).

Type A CpG oligonucleotides, like CpG ODN D32, are potent activators of IFN- $\alpha$ secretion from porcine $\mathrm{pDC}$ [14] and were employed as positive controls. While CpG induced only a few hundred units of IFN- $\alpha$ from unpurified PBMC, after magnetic cell sorting the production was increased by $\sim 10$ fold but with little differences between the different purification protocols (Figure 1B). This contrasted with pDC stimulation by FMDV O UKG 2001. As reported previously, no stimulation of PBMC or
CD172a-enriched PBMC in terms of IFN- $\alpha$ production was observed. Only the protocols employing either the CD14-depleted and subsequent CD172a-enriched PBMC or the CD3-depleted and subsequently CD4-enriched PBMC permitted the induction of IFN- $\alpha$ after stimulation with FMDV O UKG 2001 (Figure 1C). It is important to note that these responses were in the range of 100 times lower than those seen after CpG stimulation.

As the IFN- $\alpha$ level was not significantly higher when CD3-depleted/CD4-enriched PBMC were compared with the CD14-depleted/CD172a-enriched PBMC while the number of cells obtained upon enrichment was higher with the latter protocol, the CD14 depletion/ CD172a enrichment method was employed for the following experiments.

\section{pDC activation by FMDV varies between different FMDV isolates}

While testing for the capacity of different FMDV isolates to activate $\mathrm{pDC}$, we found remarkable differences, exemplified for three different FMDV isolates employed at MOIs of $1,2.5$ and $5 \mathrm{TCID}_{50}$ /cell (Figure 2A). At the lowest MOI, IFN- $\alpha$ was only detected after stimulation with O Bulgaria $1 / 91$. With the MOI of $2.5 \mathrm{TCID}_{50} /$ cell, Asia-1 Turkey 99 induced a weak response and O Bulgaria $1 / 91$ a relatively strong response. These responses were further increased when the virus dose was doubled, but O UKG 2001 remained a poor IFN- $\alpha$ inducer (Figure 2A). Considering these results, we tested more FMDV isolates for their ability to activate $\mathrm{pDC}$ at an MOI of $5 \mathrm{TCID}_{50} /$ cell (Figure 2B). The results confirmed that the ability of FMDV to activate $\mathrm{pDC}$ varies considerably, even within one serotype. Relatively strong inducers were O Bulgaria 1/91 and Asia-1 Turkey 99; weak inducers were O VietNam 7/97 and O UKG 2001, and the most inefficient inducers were $\mathrm{C} 1$ Noville, A Brazil 10/93 and A Turkey 99.

\section{Effects of a neutralizing immune serum raised against serotype $\mathrm{O}$ on strong and weak IFN- $\mathrm{a}$ inducing FMDV $\mathrm{O}$ serotype isolates}

As previous studies used purified subtype-specific antibodies [7] or high concentration of serotype-specific immune serum [8] to form FMDV immune complexes, we were interested to determine the relationship between serum concentration and IFN- $\alpha$ enhancement. Opsonisation of FMDV O UKG 2001 by a serum with a neutralization titre $(\mathrm{NT})$ of $4.1 \log _{10}$ at dilutions below 1 $\log _{10}$ did not induce pDC activation (Figure 3A). In fact, such high serum concentrations showed suppressive effects on pDC stimulation by FMDV, which were independent on the immune status of the donor animal (data not shown). They were therefore omitted in the remaining experiments. By increasing serum dilutions, 


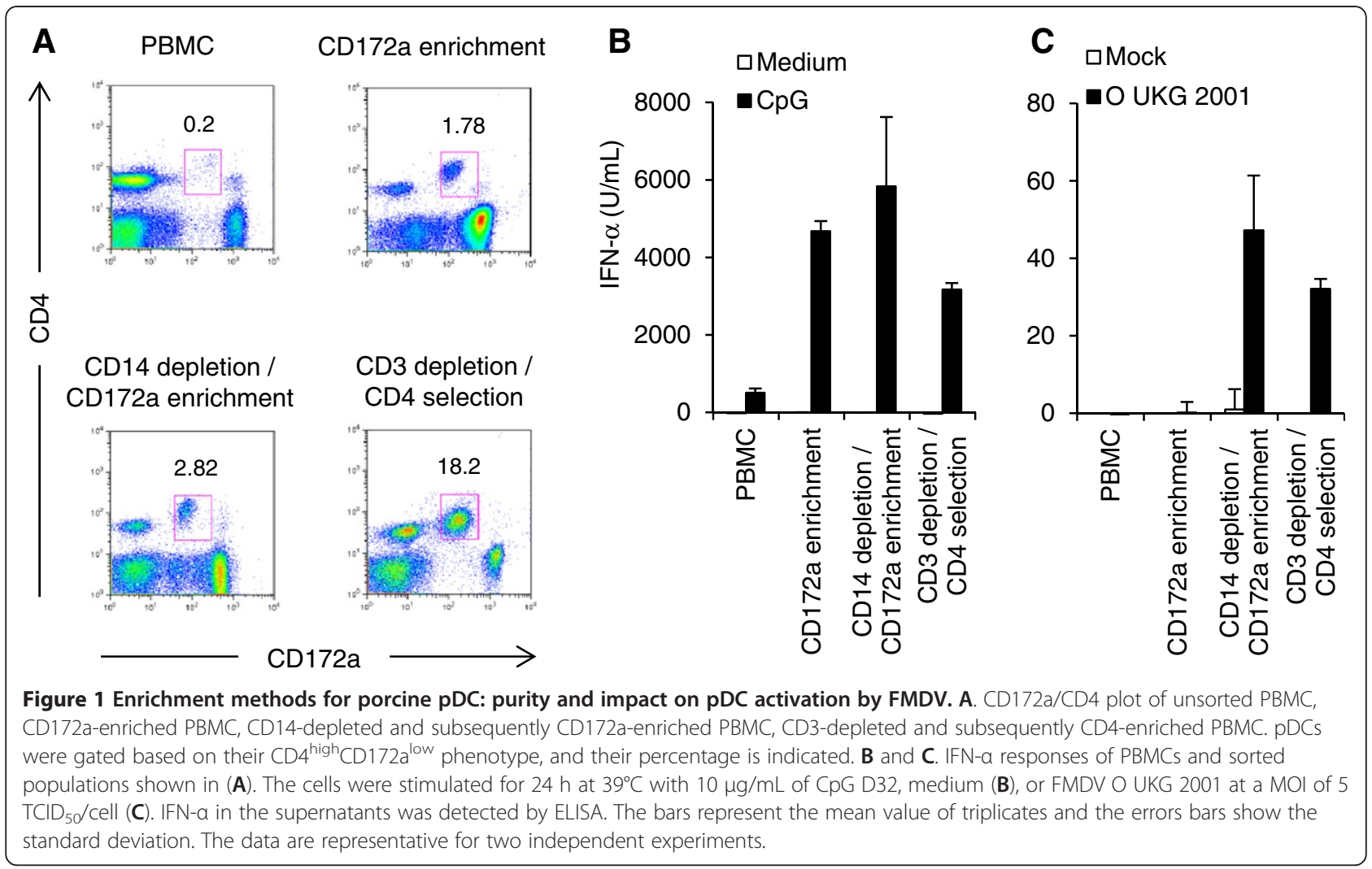

immune complexes enhanced pDC-produced IFN- $\alpha$. Even with an MOI as low as $0.5 \mathrm{TCID}_{50} /$ cell, immune complexes activated pDC (Figure 3A).

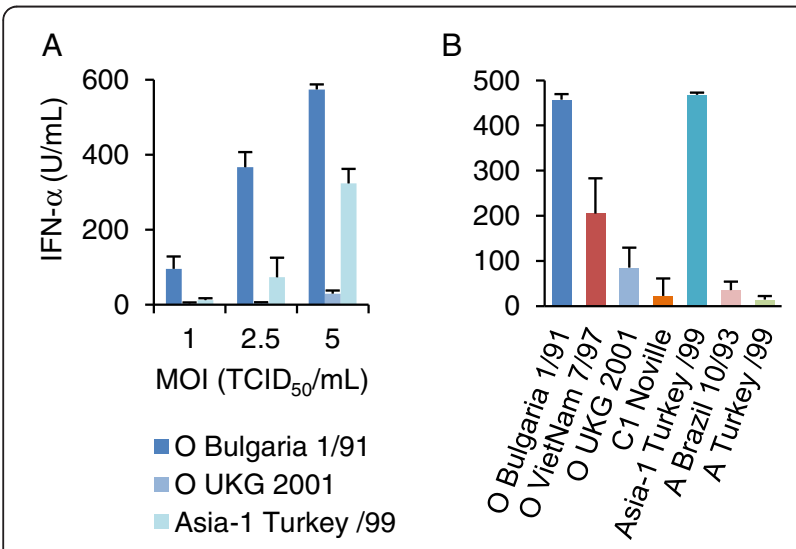

Figure 2 pDC activation by FMDV varies between different FMDV isolates. IFN-a responses of enriched pDCs (CD14-depletion/ CD172a-enrichment) stimulated by different FMDV isolates at various MOls (A) or with various FMDV isolates at an $\mathrm{MOI}$ of $5 \mathrm{TCID}_{50} /$ cell (B). After 24 h, IFN-a was measured in the supernatants by ELISA. The bars represent the mean value of triplicates and the errors bars show the standard deviation. The data are representative of three independent experiments.

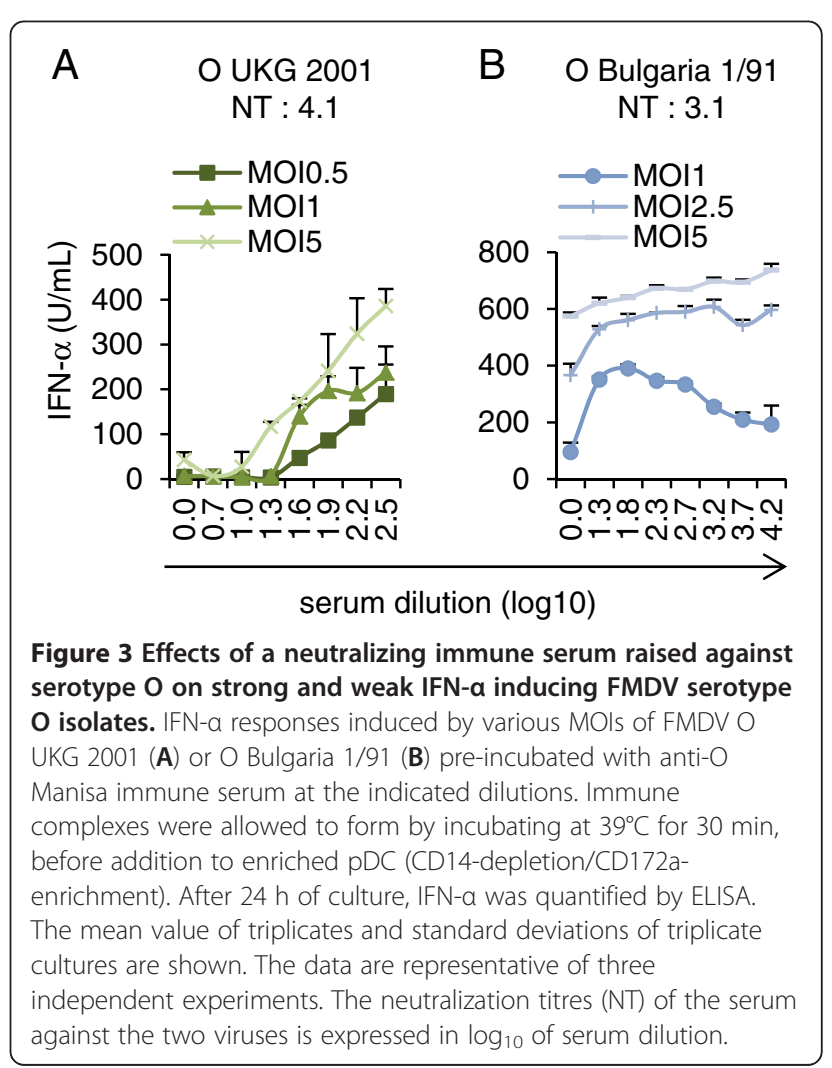


The same approach was applied for O Bulgaria 1/91, a high IFN- $\alpha$ inducing FMDV isolate (Figure 2B). The serum employed showed a NT of $3.1 \log _{10}$ against this virus. With O Bulgaria $1 / 91$ at an MOI of $1 \mathrm{TCID}_{50} / \mathrm{mL}$, immune serum enhanced pDC-derived IFN- $\alpha$ response in the expected concentration-dependent manner with maximum IFN- $\alpha$ level at a serum dilution of $1.8 \log _{10}$ and a gradual loss of the enhancing activity with further serum dilutions (Figure 3B). In contrast, when $\mathrm{O}$ Bulgaria $1 / 91$ was used at MOIs of 2.5 and $5 \mathrm{TCID}_{50} /$ cell, this serum concentration-dependent relationship was not observed (Figure 3B). In particular, with the highest virus dose a clear enhancement of IFN- $\alpha$ responses was not observed. We concluded that low MOIs have to be employed to measure antibody-dependent enhancement of IFN- $\alpha$ responses by $\mathrm{pDC}$ when high IFN- $\alpha$-inducing FMDV isolates are used.

\section{Immune complex-induced pDC activation is complement independent}

Results obtained with various serum dilutions and various FMDV isolates indicated that other serum factors than specific immunoglobulin (Ig) influenced pDC activation. For this reason a naive serum was titrated in parallel to the immune serum, and the possible impact of complement tested by heat-treatment of the sera before forming immune complexes. Figure 4 shows a representative experiment in which immune serum enhanced the IFN- $\alpha$ response induced by FMDV O UKG 2001 relative to naive serum. Nevertheless, this enhancement was only clear at a serum dilution up to $2 \log _{10}$ (Figure 4A). With heat-

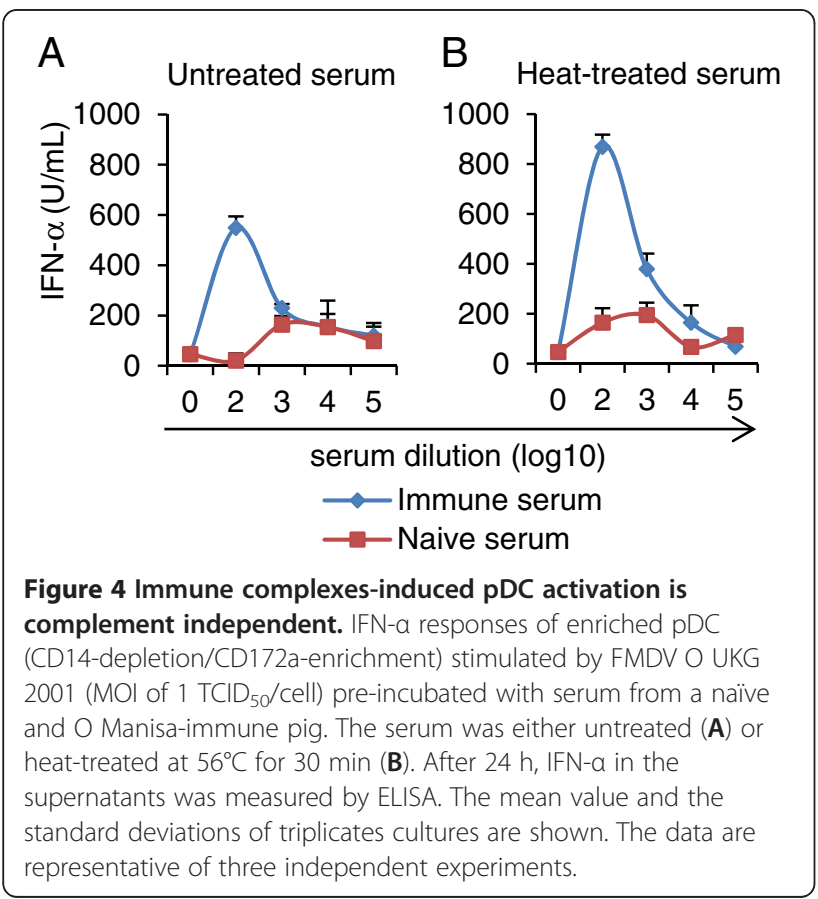

treated sera, immune complexes enhanced pDC-derived IFN- $\alpha$ were more clearly visible with statistical differences at serum dilutions up to $4 \log _{10}$. We concluded that complement is not required for the enhancement of IFN- $\alpha$ by immune-serum, and heat-labile serum factors could even have a suppressive effect. Considering this, all further experiments were performed with heat-treated serum.

\section{FMDV and immune complexes activate pDC through the TLR7 pathway}

Our previous work demonstrated that FMDV immune complex-mediated pDC activation required live virus and was associated with expression of non-structural FMDV proteins. This indicated that a virus replication cycle is initiated with formation of double stranded RNA, a potential trigger of IFN- $\alpha$ responses [5]. Although TLR7 is known to be the main sensor for RNA viruses in $\mathrm{pDC}$, it represents a receptor for single stranded RNA. We were therefore interested to determine the role played by TLR7 in sensing FMDV and FMDV immune complexes. To this end we used the immuno-regulatory sequence 661 (IRS661) representing an ODN inhibitor of TLR7 which had been previously established for human and murine immune systems [19]. We employed influenza virusstimulated pDC, a known ligand for TLR7 to test the efficiency of IRS661 to inhibit the TLR7 pathway of porcine pDC. As shown in Figure 5, 0.8-3.3 $\mu \mathrm{M}$ of IRS661 inhibited $60-70 \%$ of IFN- $\alpha$ production induced by influenza virus (Figure 5A). We next tested the ability of IRS661 at a concentration of $0.7 \mu \mathrm{M}$ to inhibit FMDV-induced pDC

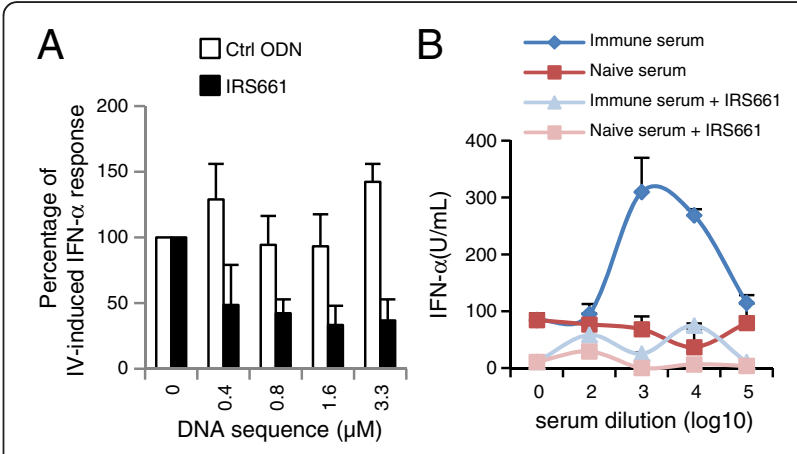

Figure 5 FMDV-induced and immune complexe-induced IFN- $a$ occurs through a TLR7-dependant pathway. A. IFN-a responses of enriched pDC (CD14-depletion/CD172a-enrichment) stimulated for $16 \mathrm{~h}$ with influenza A virus (PR8) in absence or presence of different IRS661 (TLR7 inhibitor) or control ODN (Ctrl ODN)

concentrations. B. Impact of IRS661 (0.7 $\mu \mathrm{M})$ on IFN-a responses of enriched pDC stimulated with FMDV O UKG 2001

(MOI of $1 \mathrm{TCID}_{50} /$ cell) pre-incubated for 30 min with naïve and anti-O Manisa sera. After 24 h, IFN-a was quantified by ELISA. The mean value and the standard deviations of triplicates cultures are shown. The data are representative of two independent experiments. 
activation. FMDV O UKG 2001 was not able to activate pDC in presence of IRS661. When FMDV immune complexes were employed IRS661 inhibited pDC responses by 60-80\% (Figure 5B). Similar levels of inhibition were also observed with other FMDV isolates (data not shown). The control ODN did not influence IFN- $\alpha$ production of FMDV-stimulated pDC (data not shown).

\section{PDC cultures permit to detect efficient opsonisation of FMDV in the absence of neutralization}

We were next interested to determine whether opsonising activity can also be detected in the absence of neutralizing activity. To this end, an anti-O Manisa serum was employed and tested against FMDV O UKG 2001 (NT of $4.1 \log _{10}$ ) and against A Brazil 10/93 (NT below detection limit). Interestingly, despite the inability to neutralize the A serotype virus, opsonising activity against both viruses was observed at a similar serum dilution between 2 to $4 \log _{10}$. Also the maximum IFN- $\alpha$

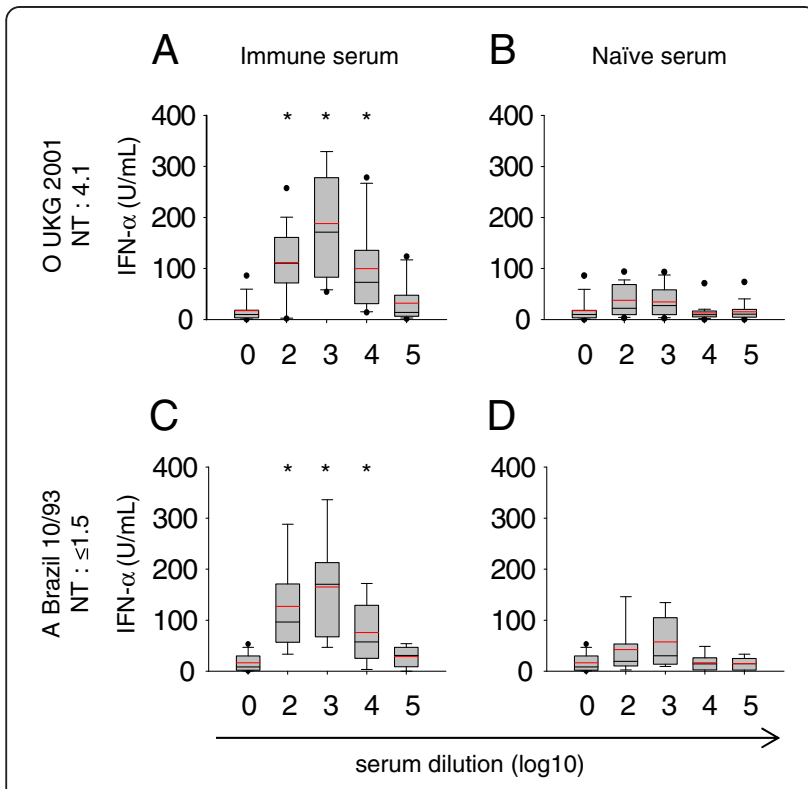

Figure 6 Opsonising activity of an anti-O Manisa serum can occur against a neutralized FMDV strain as well as a nonneutralized FMDV serotype-A strain. IFN-a responses of enriched pDC (CD14-depletion/CD172a-enrichment) stimulated with O UKG 2001 (A, B) or A Brazil 10/93 (C, D) pre-incubated with anti-O Manisa serum $(\mathbf{A}, \mathbf{C})$ or naïve serum $(\mathbf{B}, \mathbf{D})$. FMDV was used at an $\mathrm{MOI}$ of 1 $\mathrm{TCID}_{50} /$ cell. Serum was heat-treated, then diluted at the indicated concentrations and pre-incubated for $30 \mathrm{~min}$ at $39^{\circ} \mathrm{C}$ with the virus. IFN-a in supernatants was measured by ELISA. Values are shown as box plots representing of 3-5 independent experiments with each condition performed in triplicate cultures. The black line represents the median value and the red line the mean value. The error bars represent the standard deviation and the dots are outlier values $\left(5^{\text {th }} / 95^{\text {th }}\right.$ percentile). Statistical significance (Rank Sum test, $P<0.005$ ) was calculated between immune and naïve sera employed at the same dilutions and is indicated by an asterisk. The NT against the two viruses employed is indicated in $\log _{10}$ of serum dilution. production was reached at the same serum dilution of 3 $\log _{10}$ with both FMDV isolates (Figure 6A). These experiments were repeated with five different batches of pDC preparations, and the enhanced IFN- $\alpha$ responses by the immune serum compared to the effect of naive serum were consistently observed despite some variability in the height of the IFN- $\alpha$ responses (Figures $6 \mathrm{~B}$ and 6D). Similar results were also obtained with another serum sample obtained from a second vaccinated pig (data not shown). Furthermore, both sera were also able to opsonize A/Turkey/99 confirming the existence of opsonizing antibodies cross-reacting with other serotypes.

Considering these surprising results, neutralizing and opsonising activities of an anti-O Manisa serum were measured against a collection of FMDV isolates of serotypes $\mathrm{O}, \mathrm{A}, \mathrm{C}$ and Asia- 1 and the results are summarized in Table 1 as NT and opsonisation titres (OT) representing the highest serum dilution able to opsonise FMDV for enhanced IFN- $\alpha$ responses. As expected the immune serum was only able to neutralize the $\mathrm{O}$ serotype viruses O UKG 2001 and O VietNam 7/97. With these viruses we found a similar OT of 4 and $3 \log _{10}$, respectively. As shown in Figure 6C, D, non-neutralized A Brazil 10/93 could be opsonised to a titre of $4 \log _{10}$. Also FMDV A Turkey 99 and Asia-1 Turkey/99, albeit to a lesser extent, were opsonised although not being neutralized. Only, C1-Noville was neither neutralized nor opsonised by an anti-O Manisa serum. These results demonstrate that opsonisation of FMDV can occur in the absence of neutralization.

\section{Discussion}

PDC are professional sensors of viruses producing large amount of type-I IFNs. However, in particular nonenveloped viruses such as FMDV have been shown to be unable to trigger pDC activation in vitro, unless the cells are stimulated with immune complexed virus $[7,23]$. However, in the present study we demonstrate that

Table 1 Relationship between neutralization and opsonization titres

\begin{tabular}{llll}
\hline \multicolumn{3}{c}{ anti-O Manisa serum } \\
\hline Serotype & Viral strain & NT in $\mathbf{~ o g}_{\mathbf{1 0}}$ & OT $^{*}$ in $\mathbf{l o g}_{\mathbf{1 0}}$ \\
\hline Type-O & O UKG 2001 & 4.1 & 4 \\
& O VietNam 7/97 & 3.1 & 3 \\
Type-A & A Brazil 10/93 & $<1.5$ & 4 \\
& A Turkey/99 & $<1.5$ & 2 \\
Type Asia-1 & Asia-1 Turkey/99 & $<1.5$ & 2 \\
Type-C & C1-Noville & $<1.5$ & 0
\end{tabular}

* Opsonizing titres representing minimum serum dilution able to enhance IFNa responses by pDC (calculated from 3-5 independent experiments as described in Figure 6). 
FMDV can activate pDC also in absence of specific antibodies if improved purification methods are employed. We also demonstrate that both immune-complexed FMDV and uncomplexed FMDV activate pDC via TLR7. As expected, the responsiveness of pDC to FMDV is possible with more pure populations of pDC but our results also indicate that other factors could be important such as the percentage of other cell types present in the cell culture. The observation that in vivo IFN- $\alpha$ responses occur in the early innate phase of the immune response of cattle and pigs [24,25] support the idea of an early activation of pDC by FMDV in absence of specific antibodies and support the in vivo relevance of our data. In fact, in cattle it has been demonstrated that pDC are the source of early IFN- $\alpha$ responses [8].

Our study also demonstrates remarkable differences of different FMDV isolates to activate $\mathrm{pDC}$. The reason for the differences in activation of pDC could be either related to the uptake of the virus by pDC or to the function of viral genes such as the IFN antagonist $L^{\text {pro }}$, the viral proteinase. FMDV interacts with immune and nonimmune cells [26] via Arg-Gly-Asp (RGD)-dependent and RGD-independent mechanisms, after cell culture adaptation to heparin sulphate binding [27]. Certainly, the differences we observed with $\mathrm{pDC}$ are not related to a cell culture adaptation of certain FMDV isolates to heparin sulphate binding since all viruses employed in this study had a maximum of three passages. Furthermore, we have compared cell culture adapted heparinsulphate binding virus to its non-adapted original wild-type FMDV (viruses as described in [17]), and found no differences in the ability to activate pDC (data not shown). On the other hand, the leader proteinase $\mathrm{L}^{\text {pro }}$ inhibiting the type-I IFN pathway [28], is variable among serotypes [29] and could contribute to a isolate-specific FMDV counteraction on the IFN- $\alpha$ induction in pDC. The potential of some FMDV isolates to consistently induce IFN- $\alpha$ also at low MOIs would support this hypothesis. Overall, it is important to note that in comparison to the IFN- $\alpha$ levels in response to influenza viruses [21] and CpG [14], FMDV is a poor stimulator of pDC. Nevertheless, considering that IFN- $\alpha$ is known to efficiently inhibit FMDV replication in vitro and in vivo [22], it is now important to investigate the relationship of the ability of an FMDV isolate to induce IFN- $\alpha$ in vitro and to promote in vivo innate and adaptive immune responses.

Previous studies have demonstrated that antibodydependent internalization of FMDV via the FcyRII represents an important pathway to enter macrophages [5] and pDC $[7,8]$. One of the main objectives of this study was to determine the relationship between opsonisation and neutralization. Non-neutralized serotype-A and Asia-1 isolates could be efficiently opsonised using an anti-O serum while others were not, such as a serotype $\mathrm{C}$ isolate. From phylogenetic studies based on VP1 amino acid sequence, which contains the most antigenic site [30], serotypes $O$ and Asia-1 belong to the same branch [27]. Serotypes A and C are more distant, partially explaining our findings [27]. Interestingly, this opsonisation efficiently occurred with serum dilutions similar to those required for neutralization, when antigenically related viruses from the same serotypes were employed. This would indicate that the type of antibodies and their specificities is similar for both functions. However, we also found opsonisation in nonneutralizing conditions contradicting this idea. It could be speculated that opsonising activity is possible at lower avidity compared to neutralization, in which the antibody binding to the virus is in competition to virus binding to the cellular receptor. This competition can only occur indirectly for opsonisation, since the cellular FcR will bind to the Fc portion of the antibody. The observation that opsonisation can occur at similar serum dilutions with a neutralized and non-neutralized virus would favour the presence of non-neutralizing epitopes, which can be conserved between different serotypes. The answer to these questions needs to be addressed using monoclonal antibodies rather than a polyclonal serum such as in the current study.

The broad activity of opsonising antibodies is interesting in the frame of vaccine-induced immune responses. Non-neutralizing vaccine-induced antibodies could enhance innate immune responses against antigenically less related FMDV challenge strains and restrict their ability to replicate. Furthermore, other FcR-mediated functions such as virus phagocytosis and more efficient antigen presentation of viral antigens to $\mathrm{T}$ cells could be promoted. Although this is not sufficient to mediate complete protection against disease, it could limit virus replication, the severity of clinical diseases and transmission within the heard. Future studies are required to determine how such responses correlate to protection (partial or complete), and if this is the case how vaccines can be improved to promote broadly reactive antibody responses able to link innate and adaptive immunity to FMDV via opsonising antibodies.

\section{Competing interests}

The authors declare that they have no competing interests.

\section{Authors' contributions}

Conceived and design the experiments: AS, NL. Performed experiments and analysis of the data: NL, SP. Writing: NL, AS. All authors read and approved the final manuscript.

\section{Acknowledgments}

This present work was supported by EU FP7 project FMD-Disconvac (grant agreement 226556) and 3R Research Foundation Switzerland (project 11308). We thank Drs. Nigel Ferris and Satya Parida for providing some of the FMDV isolates. We also thank Heidi Gerber for mAb preparation and help 
with virus preparation as well as Andreas Michel and Hans-Peter Lüthi for regular bleeding and care of the animals.

Received: 25 May 2012 Accepted: 23 August 2012

Published: 30 August 2012

\section{References}

1. Rodriguez LL, Grubman MJ: Foot and mouth disease virus vaccines. Vaccine 2009, 27(Suppl 4):D90-94

2. McCullough KC, Bruckner L, Schaffner R, Fraefel W, Muller HK, Kihm U: Relationship between the anti-FMD virus antibody reaction as measured by different assays, and protection in vivo against challenge infection. Vet Microbiol 1992, 30:99-112

3. Robiolo B, La Torre J, Maradei E, Beascoechea CP, Perez A, Seki C, Smitsaart E, Fondevila N, Palma E, Goris N, De Clerca K, Mattion N: Confidence in indirect assessment of foot-and-mouth disease vaccine potency and vaccine matching carried out by liquid phase ELISA and virus neutralization tests. Vaccine 2010, 28:6235-6241.

4. McCullough KC, Crowther JR, Butcher RN, Carpenter WC, Brocchi E, Capucci $L$, De Simone F: Immune protection against foot-and-mouth disease virus studied using virus-neutralizing and non-neutralizing concentrations of monoclonal antibodies. Immunology 1986, 58:421-428,

5. McCullough KC, Parkinson D, Crowther JR: Opsonization-enhanced phagocytosis of foot-and-mouth disease virus. Immunology 1988, 65:187-191

6. Quattrocchi V, Langellotti C, Pappalardo JS, Olivera V, Di Giacomo S, van Rooijen N, Mongini C, Waldner C, Zamorano PI: Role of macrophages in early protective immune responses induced by two vaccines against foot and mouth disease. Antiviral Res 2011, 92:262-270.

7. Guzylack-Piriou L, Bergamin F, Gerber M, McCullough KC, Summerfield A: Plasmacytoid dendritic cell activation by foot-and-mouth disease virus requires immune complexes. Eur J Immunol 2006, 36:1674-1683.

8. Reid E, Juleff N, Gubbins S, Prentice H, Seago J, Charleston B: Bovine plasmacytoid dendritic cells are the major source of type I interferon in response to foot-and-mouth disease virus in vitro and in vivo. J Virol 2011, 85:4297-4308.

9. Summerfield A, McCullough KC: The porcine dendritic cell family. Dev Comp Immunol 2009, 33:299-309.

10. Gilliet M, Cao W, Liu YJ: Plasmacytoid dendritic cells: sensing nucleic acids in viral infection and autoimmune diseases. Nat Rev Immunol 2008, 8:594-606.

11. McKenna K, Beignon AS, Bhardwaj N: Plasmacytoid dendritic cells: linking innate and adaptive immunity. J Virol 2005, 79:17-27.

12. McCullough KC, Basta S, Knotig S, Gerber H, Schaffner R, Kim YB, Saalmuller A, Summerfield A: Intermediate stages in monocyte-macrophage differentiation modulate phenotype and susceptibility to virus infection. Immunology 1999, 98:203-212.

13. Fiebach AR, Guzylack-Piriou L, Python S, Summerfield A, Ruggli N: Classical swine fever virus $N$ (pro) limits type I interferon induction in plasmacytoid dendritic cells by interacting with interferon regulatory factor 7. J Virol 2011, 85:8002-8011.

14. Guzylack-Piriou L, Balmelli C, McCullough KC, Summerfield A: Type-A CpG oligonucleotides activate exclusively porcine natural interferonproducing cells to secrete interferon-alpha, tumour necrosis factor-alpha and interleukin-12. Immunology 2004, 112:28-37.

15. Summerfield A, Guzylack-Piriou L, Schaub A, Carrasco CP, Tache V, Charley B, McCullough KC: Porcine peripheral blood dendritic cells and natural interferon-producing cells. Immunology 2003, 110:440-449.

16. McCullough KC, Butcher R: Monoclonal antibodies against foot-andmouth disease virus $146 \mathrm{~S}$ and 125 particles. Arch Virol 1982, 74:1-9.

17. Harwood L, Gerber H, Sobrino F, Summerfield A, McCullough KC: Dendritic cell internalization of foot-and-mouth disease virus: influence of heparan sulfate binding on virus uptake and induction of the immune response. J Virol 2008, 82:6379-6394.

18. Karber G, Springer Berlin: Beitrag zur kollektiven Behandlung pharmakologischer Reihenversuche. In In Book Beitrag zur kollektiven Behandlung pharmakologischer Reihenversuche, Volume 162:; 1931:480-483.

19. Barrat FJ, Meeker T, Gregorio J, Chan JH, Uematsu S, Akira S, Chang B, Duramad O, Coffman RL: Nucleic acids of mammalian origin can act as endogenous ligands for Toll-like receptors and may promote systemic lupus erythematosus. J Exp Med 2005, 202:1131-1139.
20. Ocana-Macchi M, Bel M, Guzylack-Piriou L, Ruggli N, Liniger M, McCullough KC, Sakoda Y, Isoda N, Matrosovich M, Summerfield A: Hemagglutinindependent tropism of H5N1 avian influenza virus for human endothelial cells. J Virol 2009, 83:12947-12955.

21. Bel M, Ocana-Macchi M, Liniger M, McCullough KC, Matrosovich M, Summerfield $A$ : Efficient sensing of avian influenza viruses by porcine plasmacytoid dendritic cells. Viruses 2011, 3:312-330.

22. Charley B, Lavenant L: Characterization of blood mononuclear cells producing IFN alpha following induction by coronavirus-infected cells (porcine transmissible gastroenteritis virus). Res Immunol 1990, 141:141-151.

23. Fitzgerald-Bocarsly P: Natural interferon-alpha producing cells: the plasmacytoid dendritic cells. Biotechniques 2002, 22(Suppl:16-20):24-29.

24. Chinsangaram J, Moraes MP, Koster M, Grubman MJ: Novel viral disease control strategy: adenovirus expressing alpha interferon rapidly protects swine from foot-and-mouth disease. J Virol 2003, 77:1621-1625.

25. Alexandersen $\mathrm{S}$, Zhang Z, Donaldson Al: Aspects of the persistence of foot-and-mouth disease virus in animals-the carrier problem. Microbes Infect 2002, 4:1099-1110.

26. Summerfield A, Guzylack-Piriou L, Harwood L, McCullough KC: Innate immune responses against foot-and-mouth disease virus: current understanding and future directions. Vet Immunol Immunopathol 2009, 128:205-210.

27. Sobrino F, Saiz M, Jimenez-Clavero MA, Nunez Jl, Rosas MF, Baranowski E, Ley V: Foot-and-mouth disease virus: a long known virus, but a current threat. Vet Res 2001, 32:1-30.

28. Wang D, Fang L, Li P, Sun L, Fan J, Zhang Q, Luo R, Liu X, Li K, Chen H, Chen Z, Xiao S: The leader proteinase of foot-and-mouth disease virus negatively regulates the type I interferon pathway by acting as a viral deubiquitinase. J Virol 2011, 85:3758-3766.

29. George M, Venkataramanan R, Gurumurthy CB, Hemadri D: The non-structural leader protein gene of foot-and-mouth disease virus is highly variable between serotypes. Virus Genes 2001, 22:271-278.

30. Grubman MJ, Baxt B: Foot-and-mouth disease. Clin Microbiol Rev 2004, 17:465-493.

doi:10.1186/1297-9716-43-64

Cite this article as: Lannes et al.: Interplay of foot-and-mouth disease virus, antibodies and plasmacytoid dendritic cells: virus opsonization under non-neutralizing conditions results in enhanced interferon-alpha responses. Veterinary Research 2012 43:64.

\section{Submit your next manuscript to BioMed Central and take full advantage of:}

- Convenient online submission

- Thorough peer review

- No space constraints or color figure charges

- Immediate publication on acceptance

- Inclusion in PubMed, CAS, Scopus and Google Scholar

- Research which is freely available for redistribution 